\title{
Sistema de aquecimento solar de água: simulação e análise
}

\author{
Water solar heating systems: simulation and analysis \\ Stella Ramos Tavares ${ }^{1}$; Nádia Guimarães Sousa² \\ ${ }^{1}$ Mestre em Engenharia Química pela Universidade Federal de Uberlândia, Uberlândia, Minas Gerais, \\ Brasil. E-mail: stella_ramos_11@hotmail.com \\ 2 Professora do Curso de Engenharia Química, Universidade Federal do Triângulo Mineiro, Uberaba, \\ Minas Gerais, Brasil. E-mail: nadia.sousa@uftm.edu.br
}

RESUMO: Devido ao crescente aumento na demanda energética mundial, a busca por novas fontes de energia renováveis tem se tornado um dos principais desafios para a sociedade atual. O Brasil é um país privilegiado em termos de radiação solar, devido a isso, vem-se investindo no uso de aquecedores solares visando reduzir o consumo de energia elétrica e outras fontes de energia não renováveis. Partindo deste pressuposto, este trabalho propõe a simulação e análise do modelo matemático de sistemas de aquecimento de água utilizando coletores solares como fonte de energia. Foram avaliados dois modelos distintos: básico (sem efeito da tubulação) e estendido (com efeito da tubulação) e os cenários simulados levaram em consideração a convecção forçada (com o vento) ou convecção natural e o isolamento do sistema. Os resultados obtidos mostram que $O$ vento possui uma grande influência tanto na eficiência quanto nas temperaturas atingidas. A eficiência foi reduzida em $58 \%$, e a temperatura reduziu aproximadamente $5^{\circ} \mathrm{C}$, quando há convecção forçada. Mesmo assim, ambos os sistemas são eficientes para o aquecimento de água na região do Triângulo Mineiro, chegando a eficiência de $53 \%$ e a temperatura máxima da água do coletor de aproximadamente $80^{\circ} \mathrm{C}$.

Palavras-chave: Aquecimento; Energia solar; Modelagem matemática.

ABSTRACT: Due to the increase in world energy demand, the use of new renewable energy sources has become one of the main challenges for society. Brazil is privileged in terms of solar radiation, due to this, the use of solar heaters has been growing, aiming to reduce the consumption of non-renewable energy sources. Based on this assumption, this work proposes the simulation and analysis of the mathematical model of water heating systems using solar collectors. Two different models were evaluated: basic (no piping effect) and extended (with pipe effect) and the simulated scenarios took into account forced convection or natural convection and the isolation of the system. The results show that wind influences both, efficiency and temperatures. Efficiency has been reduced by $58 \%$, and the temperature has decreased by approximately $5^{\circ} \mathrm{C}$ when there is forced convection. Even so, both systems are efficient for water heating in the Triângulo Mineiro region, reaching efficiency of $53 \%$ and water temperature of approximately $80^{\circ} \mathrm{C}$.

Keywords: Heating; Solar energy; mathematical modelling. 


\section{INTRODUÇÃO}

O crescente aumento na demanda energética mundial é um dos grandes desafios da atualidade. Sabe-se que o uso da energia elétrica para aquecimento de água é um dos maiores responsáveis pelo seu alto consumo no país. Para reduzir esse consumo, uma alternativa viável é popularizar a utilização da energia solar para tal aquecimento.

Em termos de radiação solar, o Brasil é privilegiado. Segundo Pereira et al. (2006), a radiação solar média anual brasileira é de aproximadamente $5 \mathrm{kWhm}^{-2}$. Pensando-se na energia solar aplicada ao aquecimento têm-se um grande benefício ao utilizar aquecedores solares. Um destes está na economia no uso das energias convencionais como a eletricidade, utilizada principalmente em chuveiros elétricos.

Na Figura 1, é possível observar o impacto dos equipamentos de uma residência no gasto de energia elétrica. É nítido que o chuveiro elétrico é o que mais gasta energia. Para tentar diminuir estes gastos o uso de aquecedores solares tem demonstrado ser uma excelente alternativa, já que os investimentos totais necessários são, normalmente, inferiores aos despendidos para o aquecimento elétrico da água (CEMIG, 2012).

Figura 1 - Impacto de equipamentos e eletrodomésticos na demanda de energia.

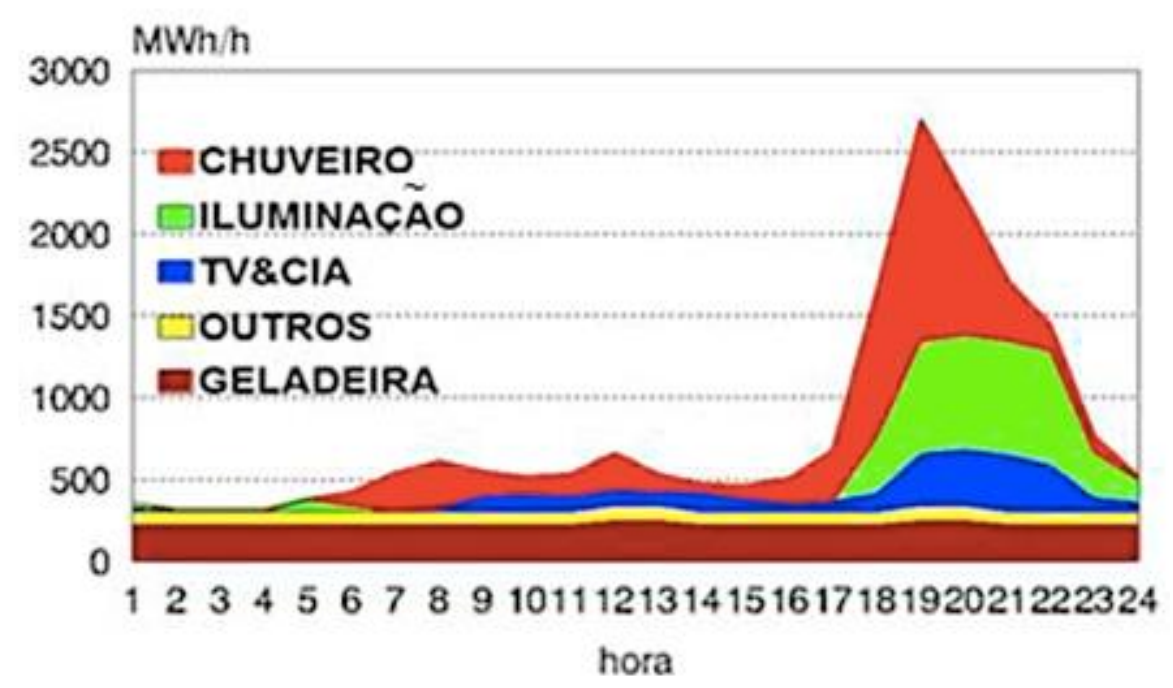

Fonte: CEMIG, 2012.

Os primeiros aquecedores solares surgiram na década de 70 , porém somente na década de 90 tiveram um aumento substancial no interesse do uso dessa tecnologia e com isso começaram a ter maiores variações de modelo, qualidade e, assim, maiores aplicações. De acordo com DASOL - ABRAVA (2015) os aquecedores são de grande importância tanto para redução do consumo de energia quanto para preservação do meio ambiente. Com a instalação do sistema de aquecimento solar, há uma economia de até $50 \%$ na conta de energia elétrica, logo a sua utilização é muito vantajosa.

Tendo em vista uma época onde a sustentabilidade é essencial, e sabendo que o país possui demanda energética solar suficiente, existem cidades que já implantaram lei para o uso de aquecedores solares. De acordo com a lei paulista $n-14.459$, de 3 de julho de 2007, desde julho de 2008 é obrigatória a instalação de aquecedores solares em novas edificações da cidade de São Paulo (SP) tais quais: residências com quatro ou mais banheiros, sistemas de aquecimento de piscinas, estabelecimentos que fazem uso 
intensivo de água quente como hospitais, hotéis, quartéis, entre outros (SÃO PAULO, 2007).

Os sistemas convencionais de aquecimento de água são simples e compostos basicamente por: reservatório de água fria, reservatório térmico, coletor solar e rede de consumo. Ao estudar o aquecimento de água por meios solares, percebe-se que, se este sistema fosse implantado em larga escala diminuiria o gasto com energia elétrica, além do que, é uma fonte de energia gratuita, renovável e por ser limpa, ajuda a preservar o meio ambiente.

De acordo com Fleck (2013), é possível, através de modelagem e simulação matemática, a geração de cenários futuros, estimação de custos e, principalmente, visualização das principais medidas preventivas a serem tomadas na execução das atividades humanas. Sendo assim, sabe-se que modelos matemáticos viabilizam e ajudam em processos futuros. Com isso, é possível analisar, por exemplo, a viabilidade de um projeto, como de um aquecedor solar, além de observar a eficiência de processos e verificar se eles valem a pena ser feitos.

Mesmo sendo um assunto muito abordado em estudos, com diferentes enfoques, avaliar novas possibilidades de configurações e melhorias no sistema de aquecimento, visando um melhor aproveitamento da fonte de energia solar é de grande valia do ponto de vista econômico e ambiental. Partindo desse pressuposto o foco principal deste trabalho é o estudo de sistemas de aquecimento solar, com a finalidade de aquecimento de água. Para isso, tem-se a análise e simulação do modelo matemático de duas configurações distintas de aquecedores solares: o modelo matemático básico, que não leva em consideração o efeito da tubulação no aquecimento da água e o modelo matemático estendido, em que esse efeito é considerado. Avalia-se também efeito da velocidade do vento (convecção forçada) e do isolamento do sistema na temperatura final da água.

\section{REVISÃO DA LITERATURA}

\section{Modelagem matemática e simulação de processos}

O interesse industrial em técnicas e pacotes computacionais para a modelagem e simulação de processos tem crescido nos últimos anos. Esse crescimento é influenciado por vários fatores, tais como os econômicos, ambientais e de segurança e risco. Portanto, análise de todo processo através do seu modelo matemático visando à melhoria tem ganhado cada vez mais espaço (SECCHI, 2002).

Devido aos avanços computacionais o uso de modelos matemáticos e simuladores de processos químicos tem transposto novas fronteiras além das tradicionais aplicações no dimensionamento de equipamentos e na solução de problemas operacionais de processos químicos. Atualmente, são largamente utilizados em aplicações de treinamento de operadores, no auxílio de programação e planejamento de produção e na melhoria e otimização de unidades de processos químicos (REJOWSKI JUNIOR, 2018).

Os modelos matemáticos bem feitos podem auxiliar na de tomada de decisões em relação a como cada equipamento deve operar no consumo/geração de vapor e de fontes alternativas de combustível e no gerenciamento de geração/consumo de energia elétrica para atender a uma dada demanda energética. (REJOWSKI JUNIOR, 2018).

A obtenção do modelo matemático de processos químicos é feita através da aplicação do princípio básico de conservação de massa e/ou energia no sistema 
estudado. A sistematização da construção do modelo é realizada, de forma geral, pela definição do volume de controle (V.C.) em que será aplicado o princípio básico, ou seja, em qual equipamento, tubulação, fase distinta serão realizados o balanço de massa e energia. As equações 1 e 2 representam a forma geral de balanço de massa e energia aplicado à um volume de controle genérico, com $n_{e}$ correntes de entrada e $n_{s}$ correntes de saída, respectivamente.

$$
\begin{aligned}
& \frac{\mathrm{dm}_{\mathrm{T}}}{\mathrm{dt}}=\sum_{\mathrm{i}=1}^{\mathrm{n}_{\mathrm{e}}} \dot{\mathrm{m}}_{\mathrm{T}_{\mathrm{ei}}}-\sum_{\mathrm{i}=1}^{\mathrm{n}_{\mathrm{s}}} \dot{\mathrm{m}}_{\mathrm{T}_{\mathrm{si}}} \\
& \frac{\mathrm{dE}}{\mathrm{dt}}=\sum_{\mathrm{i}=1}^{\mathrm{n}_{\mathrm{e}}} \dot{\mathrm{E}}_{\mathrm{e}_{\mathrm{i}}}-\sum_{\mathrm{i}=1}^{\mathrm{n}_{\mathrm{s}}} \dot{\mathrm{E}}_{\mathrm{s}_{\mathrm{i}}}+\dot{\mathrm{Q}}+\dot{\mathrm{W}}+\dot{\mathrm{E}}_{\mathrm{g}}
\end{aligned}
$$

Entretanto, no processo de obtenção dos modelos matemáticos, é importante destacar que à medida que um processo torna-se mais complexo e mais rigorosa for a sua descrição, o conjunto de equações resultantes será mais difícil de tratar. Ainda que elas possam ser resolvidas, é necessário utilizar de hipóteses simplificadoras para reduzir as equações a um conjunto menos complexo que, para propósitos práticos, resultará em soluções dentro da precisão dos dados disponíveis (SECCHI, 2002).

\section{Aquecedores solares e modelagem matemática e simulação}

O uso de aquecedores solares tem tido um rápido crescimento em sua aplicação motivado por questões importantes como a crise energética mundial. Como exemplo do uso dessa tecnologia pode ser citado o trabalho de Russi (2012) que buscou a criação de residências mais sustentáveis. Nesse trabalho foi proposto um projeto do modelo utilizando a simulação matemática de um sistema combinado, em que a energia solar foi aproveitada tanto para aquecimento de água, quanto para condicionamento térmico de edificação nos períodos frios. Os resultados obtidos mostraram que é possível obter bons resultados mesmo em regiões frias como o sul do Brasil.

O estudo de Dacol (2012) verificou a viabilidade de instalação e utilização de coletores solares para aquecimento de água de chuveiro elétrico. Para isto foram utilizadas duas residências, uma com aquecimento solar e uma sem, sendo que ambas possuíam a mesma quantidade de moradores e mesmo tempo de banho. Com os modelos e os dados analisados foi possível a comparação entre as duas residências e verificou-se que é viável a instalação de coletores solares para o aquecimento de água, pois isso contribuiu para um menor consumo energético.

Com o objetivo de estudar coletores solares feitos de materiais alternativos, Janjai et al. (2000) desenvolveram um modelo para a simulação de um coletor solar de grande área feito de plástico. Para que o modelo proposto fosse aprovado foram realizados testes para verificação do valor da temperatura da saída de água, ao se observar que ela estava de acordo com o esperado, foi então validado para utilização. Assim os resultados obtidos através da simulação indicaram que parâmetros como o comprimento do coletor, afetam significativamente a temperatura de saída de água e que esta temperatura não é sensível à velocidade do vento. 
Utilizando a modelagem matemática a parâmetros concentrados, Kicsiny et al. (2014) propuseram quatro modelos matemáticos, sendo dois lineares - básico e estendido e dois não lineares - básico e estendido. Sabendo que o modelo estendido leva em consideração os efeitos causados pela tubulação, e o básico não, foram feitas simulações para os modelos lineares (básico e estendido). Com essas simulações foi possível observar que o modelo estendido era melhor quando comparado aos resultados da simulação do modelo básico. Vale ressaltar que, tanto o modelo básico quanto o estendido não simulam muito bem os coletores solares, pois existem diversas perturbações que não são levadas em consideração, como a sombra causada pelas nuvens.

Com a finalidade avaliar as barreiras existentes na implementação dos sistemas de aquecimento solar, Wang et al. (2015) realizaram um estudo sobre as forças motrizes para a expansão do mercado térmico solar que são: o preço do aquecedor solar, incentivos financeiros oferecidos em alguns locais para encorajar consumidores, entre outros. O resultado desse trabalho ajuda na identificação da situação atual e dos potenciais problemas existentes para aumento do uso de aquecedores solares, e também dá direções futuras em relação à pesquisa, desenvolvimento e aplicação prática de tecnologias de aquecimento solar em edifícios.

\section{MATERIAIS E MÉTODOS}

Este trabalho possui natureza teórico-computacional e utiliza o software livre, Scilab® - solucionador Isoda do pacote ODEPACK - para realizar as simulações computacionais do modelo matemático dos sistemas estudados. Tem-se a análise e simulação do modelo matemático de duas configurações distintas de aquecedores solares: o modelo matemático básico e o modelo matemático estendido. Avalia-se também efeito da velocidade do vento (convecção natural e forçada) e do isolamento do sistema na temperatura final da água. Com os resultados gerados pela simulação dos modelos matemáticos, é possível analisar qual a melhor configuração e como essas variáveis afetam o sistema de aquecimento. A Figura 2 apresenta o esquema da metodologia utilizada neste trabalho.

Figura 2 - Esquema da metodologia utilizada no trabalho.

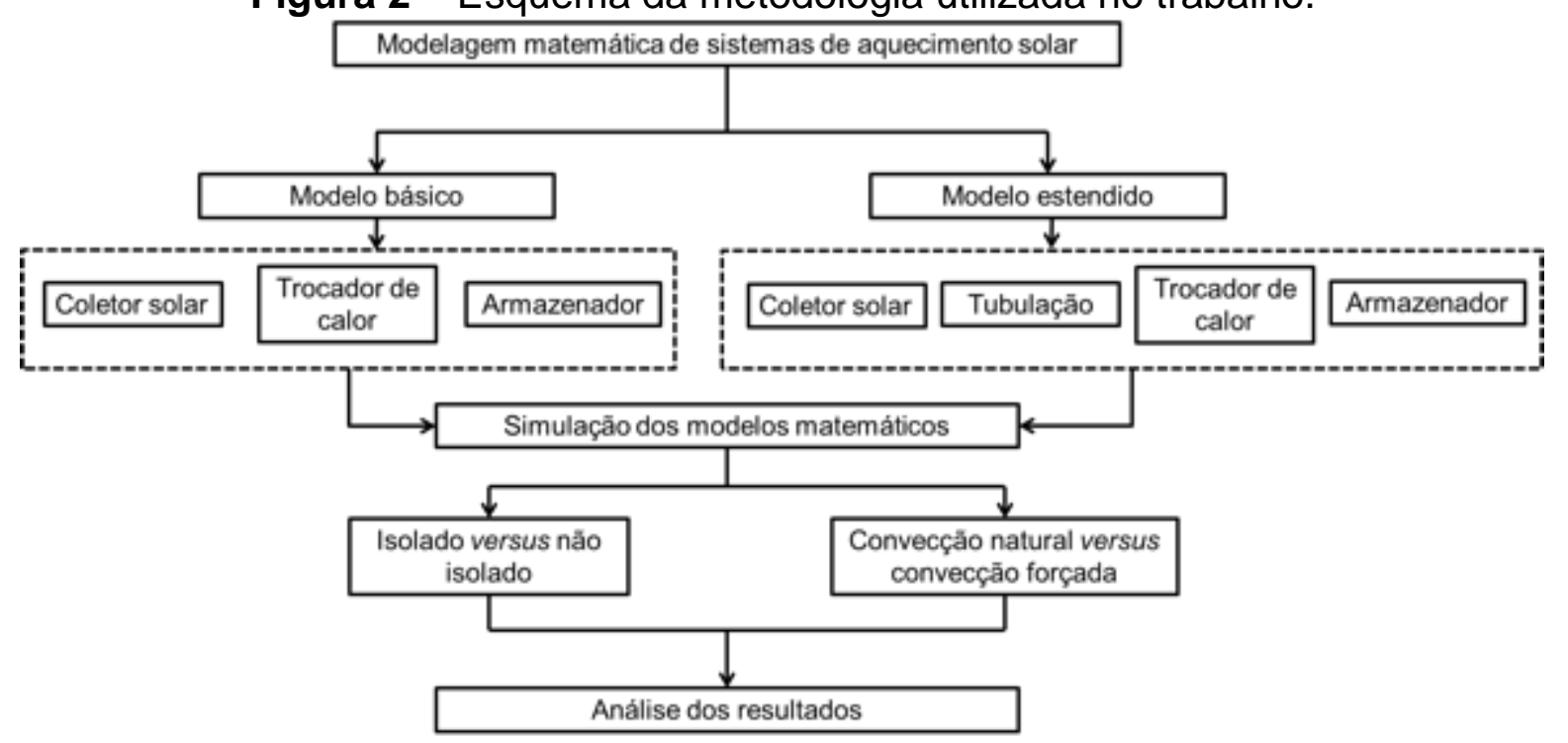


A obtenção do modelo matemático é realizada, pela definição do volume de controle (V.C.) em que será aplicado o princípio básico de conservação de energia (equação 2). Para o modelo básico, o princípio de conservação de energia, é aplicado ao coletor solar, trocador de calor e armazenador. Já para o modelo estendido, o balanço de energia é realizado no coletor solar, tubulações e armazenador.

Assim, o modelo básico possui as seguintes temperaturas como variáveis de estado: coletor $\left(T_{c}\right)$, armazenador $\left(T_{s}\right)$, parte quente do trocador de calor $\left(T_{h h}\right)$ e a parte fria do trocador de calor $\left(T_{h c}\right)$. O modelo estendido não apresenta $T_{h h}$ e $T_{h c}$ como variáveis de estado, porém ele possui as equações que levam em conta os efeitos da tubulação como: temperatura do tubo entre a saída do coletor e o trocador de calor $\left(T_{p c 1}\right)$, a temperatura do tubo entre a saída do trocador de calor e a entrada do coletor $\left(T_{p c 2}\right)$, a temperatura do tubo entre a saída do armazenador e o trocador de calor $\left(T_{p s 2}\right)$.

Como hipóteses simplificadoras foi considerado que as temperaturas $\left(T_{c}, T_{s}, T_{h h}, T_{h c}, T_{p c 1}, T_{p s 1}, T_{p c 2}, T_{p s 2}\right)$ são homogêneas e variam com o tempo, mistura perfeita, e que as perdas por radiação são desprezíveis. O modelo matemático utilizado foi baseado no trabalho de Kicsiny et al. (2014) sendo que foram implementadas algumas modificações com a finalidade de se aproximar ao máximo a um sistema de aquecimento real.

\section{Modelagem matemática}

Os sistemas estudados estão mostrados nas Figuras 3 e 4 . A Figura 3 representa o esquema para o sistema básico e a Figura 4 representa sistema estendido. Considerar os efeitos das tubulações na obtenção do modelo matemático aumenta sua complexidade, mas permite que sejam consideradas as trocas térmicas que ocorrem entre as tubulações e suas vizinhanças.

Figura 3 - Representação do aquecedor solar do modelo linear básico.

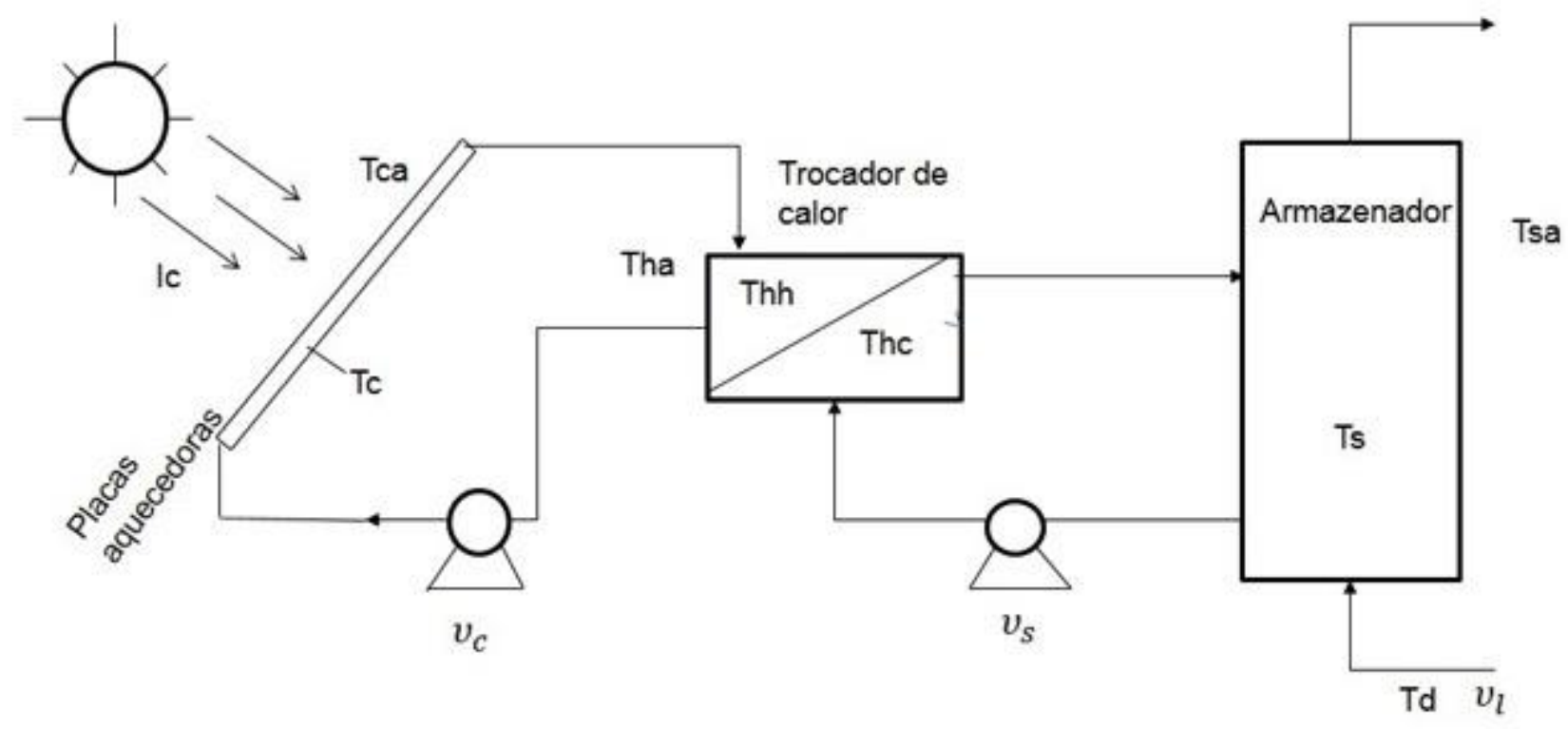

Fonte: Kicsiny et al., 2014. 
Figura 4 - Representação do aquecedor solar do modelo estendido.

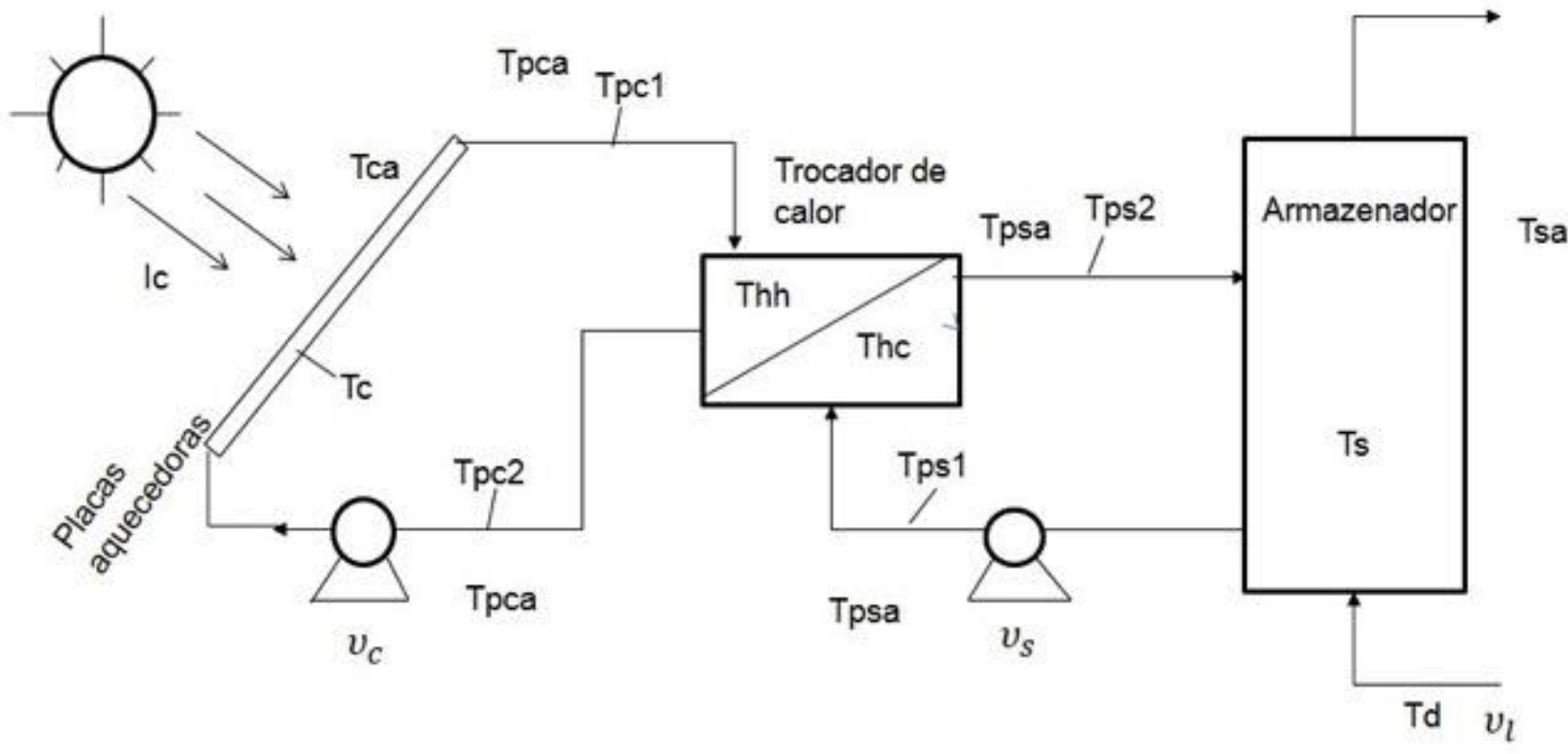

Fonte: Kicsiny et al., 2014.

Após a aplicação do princípio de conservação de energia, equação 2, às configurações anteriormente apresentadas, obteve-se os dois modelos matemáticos. $\mathrm{O}$ modelo matemático básico é descrito pelas equações 3 a 6 .

$$
\begin{aligned}
& \frac{d T_{c}}{d t}=\frac{A_{c} \eta_{0}}{\rho_{c} c_{c} V_{c}} I_{c}+\frac{U_{L b} A_{c}}{\rho_{c} c_{c} V_{c}}\left(T_{c a}-T_{c}\right)+\frac{v_{c}}{V_{c}}\left(T_{h h}-T_{c}\right) \\
& \frac{d T_{s}}{d t}=\frac{v_{l}}{V_{s}}\left(T_{d}-T_{s}\right)+\frac{v_{s}}{V_{s}}\left(T_{h c}-T_{s}\right)+\frac{A_{s} k_{s}}{\rho_{s} c_{s} V_{s}}\left(T_{s a}-T_{s}\right) \\
& \frac{d T_{h h}}{d t}=\frac{\rho_{c} c_{c} V_{c}}{\frac{c_{h} m_{h}}{2}+\rho_{c} c_{c} \frac{V_{h}}{2}}\left(T_{c}-T_{h h}\right)+\frac{\varepsilon k_{h} A_{h}}{\frac{c_{h} m_{h}}{2}+\rho_{c} c_{c} \frac{V_{h}}{2}}\left(T_{h c}-T_{h h}\right)+ \\
& \quad+\frac{\frac{A_{a} k_{h}}{2}}{\frac{c_{h} m_{h}}{2}+\rho_{c} c_{c} \frac{V_{h}}{2}}\left(T_{h a}-T_{h h}\right) \\
& \frac{d T_{h c}}{d t}=\frac{\rho_{s} c_{s} V_{s}}{\frac{c_{h} m_{h}}{2}+\rho_{s} c_{s} \frac{V_{h}}{2}}\left(T_{s}-T_{h c}\right)+\frac{\varepsilon k_{h} A_{h}}{\frac{c_{h} m_{h}}{2}+\rho_{s} c_{s} \frac{V_{h}}{2}}\left(T_{h h}-T_{h c}\right)+ \\
& \quad+\frac{A_{a} k_{h}}{2} \\
& \frac{c_{h} m_{h}}{2}+\rho_{s} c_{s} \frac{V_{h}}{2}
\end{aligned}
$$


O modelo matemático estendido é representado pelas equações 7 a 12 .

$$
\begin{aligned}
& \frac{d T_{c}}{d t}=\frac{A_{c} \eta_{0}}{\rho_{c} c_{c} V_{c}} I_{c}+\frac{U_{L e} A_{c}}{\rho_{c} c_{c} V_{c}}\left(T_{c a}-T_{c}\right)+\frac{v_{c}}{V_{c}}\left(T_{p c 2}-T_{c}\right) \\
& \frac{d T_{s}}{d t}=\frac{v_{l}}{V_{s}}\left(T_{d}-T_{s}\right)+\frac{v_{s}}{V_{s}}\left(T_{p s 2}-T_{s}\right)+\frac{A_{s} k_{s}}{\rho_{s} c_{s} V_{s}}\left(T_{s a}-T_{s}\right) \\
& \frac{d T_{p c 1}}{d t}=\frac{v_{c}}{V_{p c}}\left(T_{c}-T_{p c 1}\right)+\frac{L_{p c} k_{p c}}{\rho_{c} c_{c} V_{p c}}\left(T_{p c a}-T_{p c 1}\right) \\
& \frac{d T_{p c 2}}{d t}=\frac{v_{c}}{V_{p c}}\left(T_{p c 1}-T_{p c 2}\right)+\Phi \frac{\rho_{s} c_{s} v_{s}}{\rho_{c} c_{c} V_{p c}}\left(T_{p s 1}-T_{p c 1}\right)+\frac{L_{p c} k_{p c}}{\rho_{c} c_{c} V_{p c}}\left(T_{p c a}-T_{p c 2}\right) \\
& \frac{d T_{p s 1}}{d t}=\frac{v_{s}}{V_{p s}}\left(T_{s}-T_{p s 1}\right)+\frac{L_{p s} k_{p s}}{\rho_{s} c_{s} V_{p s}}\left(T_{p s a}-T_{p s 1}\right) \\
& \frac{d T_{p s 2}}{d t}=\frac{v_{s}}{V_{p s}}\left(T_{p s 1}-T_{p s 2}\right)+\Phi \frac{v_{s}}{V_{p s}}\left(T_{p c 1}-T_{p s 1}\right)+\frac{L_{p s} k_{p s}}{\rho_{s} c_{s} V_{p s}}\left(T_{p s a}-T_{p s 2}\right)
\end{aligned}
$$

As equações para o trocador de calor, equação 5 e 6, são substituídas por equações obtidas a partir do método da efetividade - NUT. A equação 13 representa a temperatura da parte quente do trocador de calor do modelo estendido e na equação 14 é visualizada a temperatura da parte fria.

$$
\begin{aligned}
& \mathrm{T}_{\mathrm{hh}}=\mathrm{T}_{\mathrm{pc} 1}-\Phi\left(\mathrm{T}_{\mathrm{pc} 1}-\mathrm{T}_{\mathrm{ps} 1}\right) \frac{\rho_{\mathrm{s}} \mathrm{c}_{\mathrm{s}} \mathrm{v}_{\mathrm{s}}}{\rho_{\mathrm{c}} \mathrm{c}_{\mathrm{c}} \mathrm{v}_{\mathrm{c}}} \\
& \mathrm{T}_{\mathrm{hc}}=\Phi\left(\mathrm{T}_{\mathrm{pc} 1}-\mathrm{T}_{\mathrm{ps} 1}\right)+\mathrm{T}_{\mathrm{ps} 1}
\end{aligned}
$$

A maioria dos trabalhos encontrados na literatura adotam simplificações para facilitar a resolução dos modelos obtidos. Visando uma modelagem mais real, a qual represente com mais fidelidade um aquecedor solar na prática, foi implementada algumas modificações nos modelos matemáticos propostos por Kicsiny et al. (2014). A seguir serão apresentas essas modificações.

\section{Modelo modificado}

Para os coeficientes de perda de calor, $U_{L b}$ e o $U_{L e}$, foram utilizadas equações que representem a convecção natural e forçada. Diferentemente do trabalho de Kicsiny et al. 
(2014) que utilizou valores fixos, não levando em consideração como mudanças ambientais afetam na temperatura da água. Assim, estes coeficientes são iguais para ambos os modelos. Para a convecção natural tem-se a equação 15 (INCROPERA et al., 2011).

$$
\mathrm{U}_{\mathrm{Le}}=\mathrm{U}_{\mathrm{Lb}}=\frac{\mathrm{k}_{\mathrm{w}} \mathrm{C}(\mathrm{GrPr})^{\mathrm{n}}}{\mathrm{L}}
$$

Em que: $L[m]$ é o comprimento da placa e Gr é o número de Grashof e este está representado na equação 16.

$$
\mathrm{Gr}=\frac{\beta g\left(\mathrm{~T}_{\mathrm{c}}-\mathrm{T}_{\mathrm{ca}}\right) \mathrm{L}^{3}}{\mathrm{v}^{2}}
$$

Em que: $v\left[m^{2} s^{-1}\right]$ é a viscosidade cinemática e $\beta$ é o coeficiente de expansão volumétrica térmica e este é visualizado na equação 17 (YUNUS, 2009 apud GOMES FILHO, 2013).

$$
\beta=\frac{2}{\mathrm{~T}_{\mathrm{c}}+\mathrm{T}_{\mathrm{ca}}}
$$

Quando se considera convecção forçada, o efeito causado pelo vento é adicionado, com isso, para os coeficientes de perda de calor, $U_{L b}$ e o $U_{L e}$, foram utilizadas equações que representem esse tipo de convecção. Assim, estes coeficientes são iguais para ambos os modelos, e estão representados na equação 18 (PALYVOS, 2008).

$$
\mathrm{U}_{\mathrm{Lb}}=\mathrm{U}_{\mathrm{Le}}=7,13\left(\mathrm{vel}^{0,78}\right)+5,35 \mathrm{e}^{-0,6 \mathrm{vel}}
$$

Em que: vel $\left[\mathrm{ms}^{-1}\right]$ é a velocidade do vento. $\mathrm{O}$ cálculo da eficiência de aquecimento é dado pela equação 19.

$$
\mathrm{N}_{\mathrm{a}}=\frac{\rho_{\mathrm{s}} \mathrm{c}_{\mathrm{s}} \mathrm{v}_{\mathrm{s}}\left(\mathrm{T}_{\mathrm{sm}}-\mathrm{T}_{\mathrm{sam}}\right)}{\mathrm{A}_{\mathrm{c}} \mathrm{I}_{\mathrm{cm}}}
$$

Em que: $\mathrm{T}_{\mathrm{sm}}[K]$ é a temperatura média do armazenador, $\mathrm{T}_{\text {sam }}[K]$ é a temperatura ambiente média do armazenador e $\mathrm{I}_{\mathrm{cm}}\left[\mathrm{Wm}^{-2}\right]$ é a irradiação solar média calculada para o mês janeiro de 2015.

\section{Dados do processo}

Para o modelo proposto foram utilizadas as seguintes hipóteses simplificadoras: irradiação considerada é a total $\left(I_{c}\right)$ e esta é perpendicular ao coletor; o coletor, as duas partes do trocador de calor, o armazenador e as tubulações, possuem uma mistura perfeita, sendo assim, suas temperaturas são homogêneas e para o sistema isolado, considerou-se que os coeficientes de perda de calor para o ambiente do trocador de calor, das tubulações do coletor e do armazenador são zero, pois estes estão isolados. 
Tendo isso em mente, os valores de $I_{c}$, velocidade do vento $(\mathrm{vel})$ e todas as temperaturas ambientes foram retirados do Inmet (2015) para a cidade de Uberlândia MG, e os outros parâmetros utilizados tanto para o modelo básico quanto para o modelo estendido estão disponíveis na Tabela 1.

Tabela 1 - Parâmetros para os modelos básico e estendido

\begin{tabular}{|c|c|c|c|}
\hline Parâmetro & Valor & Parâmetro & Valor \\
\hline$\overline{A_{c}}$ & $33,3 \mathrm{~m}^{2}$ & $\overline{A_{a}}$ & $0,24 \mathrm{~m}^{2}$ \\
\hline$\rho_{s}$ & $1000 \mathrm{kgm}^{-3}$ & $c_{s}$ & $4200 \mathrm{Jkg}^{-1}$ \\
\hline$\rho_{c}$ & $1034 \mathrm{kgm}^{-3}$ & $V_{c}$ & $0,027 \mathrm{~m}^{3}$ \\
\hline$V_{s}$ & $2 \mathrm{~m}^{3}$ & $\eta_{0}$ & 0,74 \\
\hline$T_{d}$ & $298,15 K$ & $\boldsymbol{k}_{s}$ & $1 W^{-2} K^{-1}$ \\
\hline$m_{h}$ & $37 \mathrm{~kg}$ & $V_{p c}$ & $0,111 \mathrm{~m}^{3}$ \\
\hline$L_{p s}$ & $115 \mathrm{~m}$ & $V_{p s}$ & $0,158 \mathrm{~m}^{3}$ \\
\hline$L$ & $2 m$ & $v$ & $15,73 \cdot 10^{-3} \mathrm{~m}^{2} \mathrm{~s}^{-1}$ \\
\hline$n$ & $1 / 3$ & $v_{c}$ & $2,72 \cdot 10^{-4} \mathrm{~m}^{3} \mathrm{~s}^{-1}$ \\
\hline$c_{c}$ & $3623 \mathrm{Jkg}^{-1} \mathrm{~K}^{-1}$ & $\mathrm{Pr}$ & 0,707 \\
\hline$c_{h}$ & $464,8 \mathrm{Jkg}^{-1} \mathrm{~K}^{-1}$ & $\varepsilon \boldsymbol{k}_{h}$ & $2461,5 \mathrm{Wm}^{-2}$ \\
\hline$v_{l}$ & $1.10^{-4} \mathrm{~m}^{3} \mathrm{~s}^{-1}$ & $L_{p c}$ & $80 \mathrm{~m}$ \\
\hline$A_{s}$ & $4 m^{2}$ & C & 0,15 \\
\hline$P r$ & 0,707 & $v_{s}$ & $17,5.10^{-4} \mathrm{~m}^{3} \mathrm{~s}^{-1}$ \\
\hline$V_{h}$ & $0,005 \mathrm{~m}^{3}$ & $A_{h}$ & $2 m^{2}$ \\
\hline$\Phi$ & 0,89 & $\boldsymbol{k}_{p s}$ & $0,16 \mathrm{~W} / \mathrm{m}^{2} \mathrm{~K}$ \\
\hline $\boldsymbol{k}_{h}$ & $5 \mathrm{~W} / \mathrm{m}^{2} \mathrm{~K}$ & $k_{p c}$ & $0,22 \mathrm{~W} / \mathrm{m}^{2} \mathrm{~K}$ \\
\hline
\end{tabular}

Fonte: Kicsiny et al., 2012; 2014.

\section{RESULTADOS E DISCUSSÃO} estendido.

Serão analisados dois casos que serão divididos pelos modelos básico e

Caso 1: representação do modelo básico onde estão representadas as equações 3 a 6.

$\checkmark$ Caso 2: representação do modelo estendido onde estão representadas as equações 7 a 12.

Com os parâmetros definidos foram feitas as simulações utilizando o software livre Scilab®. A comparação da temperatura da água para os dois modelos analisados, levando em conta o efeito do vento, é mostrado na Figura 5. Analisando os quatro modelos o que apresentou melhores resultados, para ambos os casos, foi o estendido isolado. Isso ocorre, pois, neste modelo, foi levado em consideração o efeito das tubulações, ou seja, foi considerada a capacidade calorífica dos tubos e estando isolados, eles mantêm calor por mais tempo e consegue chegar a uma temperatura maior. 
Figura 5 - Temperatura do armazenador: (a) desconsiderando o efeito causado pelo vento. (b) considerando o efeito causado pelo vento.

(a)

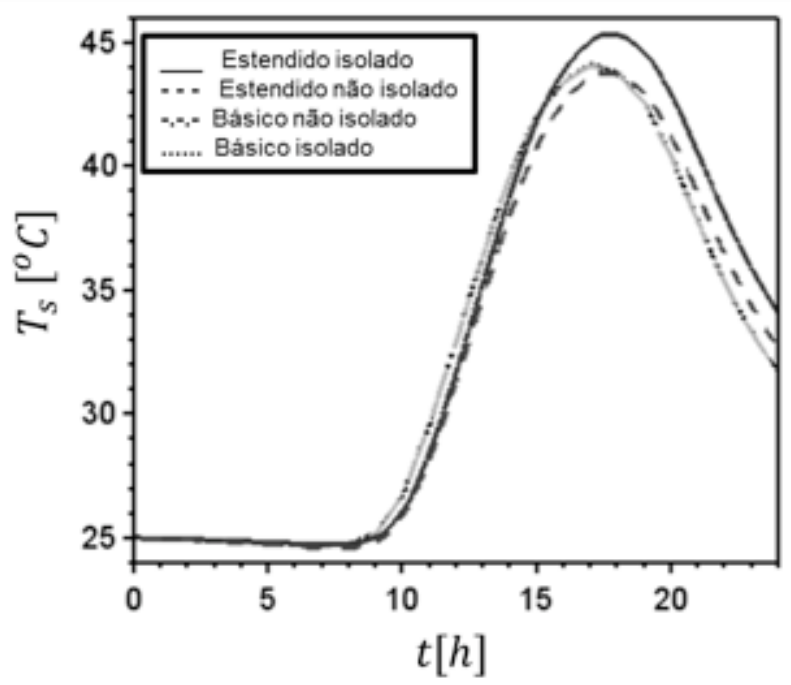

(b)

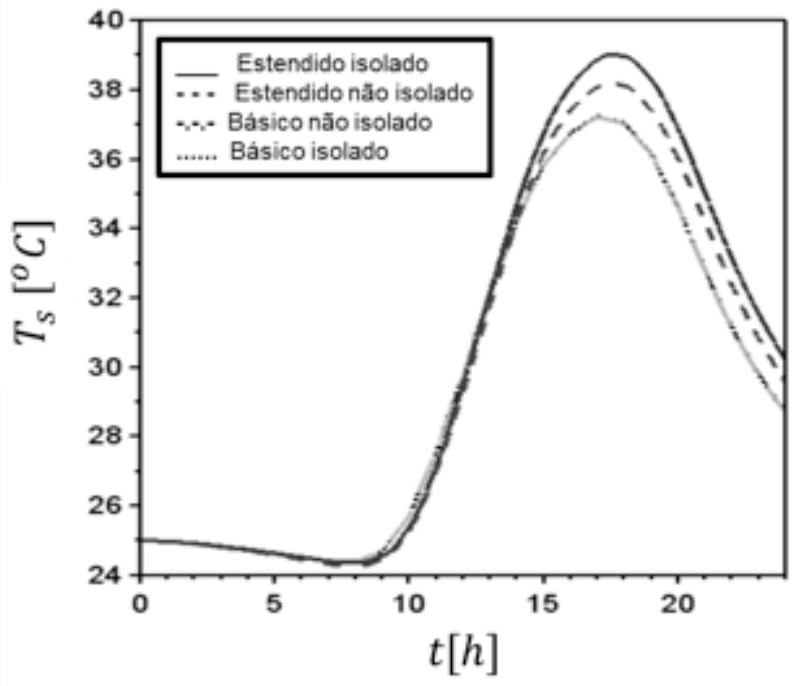

O efeito da capacidade calorífera da tubulação também afeta o tempo de aquecimento; no modelo estendido o armazenador começa a aquecer um pouco depois, mas mantém a temperatura por mais tempo. No modelo básico, essa diferença quase não é percebida. Utilizando a equação 19 e os valores das temperaturas do armazenador, Figura 5, foi possível encontrar a eficiência do aquecimento da água para os modelos propostos. Esses valores estão dispostos na Tabela 2.

Tabela 2 - Eficiência do armazenador.

\begin{tabular}{l|ll|ll}
\hline & \multicolumn{2}{|c|}{ Isolado } & \multicolumn{2}{c}{ Não isolado } \\
\hline & Com vento & Sem vento & Com vento & Sem vento \\
Básico & 0,2429 & 0,4705 & 0,2424 & 0,4693 \\
Estendido & 0,3090 & 0,5300 & 0,2788 & 0,4724 \\
\hline
\end{tabular}

É visto que, pela análise da Tabela 2 e pela Figura 5, o vento possui uma grande influência tanto na eficiência quanto nas temperaturas atingidas. Para o modelo estendido e isolado, que obteve os melhores resultados, a eficiência foi reduzida em $58 \%$, e a temperatura reduziu aproximadamente $5^{\circ} \mathrm{C}$. Essa diferença pode ser explicada, pois 0 coletor tem mais dificuldade em manter a temperatura devido à convecção forçada e com isso a temperatura do armazenador se torna menor.

Porém, mesmo com tais dificuldades o modelo estendido isolado continua sendo o melhor, pois mesmo aquecendo menos, a capacidade calorífica da tubulação isolada faz com que ele a mantenha calor por mais tempo atingindo maiores temperaturas máxima e final.

O comportamento das demais temperaturas para o caso 1 é mostrado na Figura 6. Neste caso, pode-se observar que, para o modelo básico a diferença entre as simulações do isolado e do não isolado são mínimas. Só é possível observar tal diferença ao analisar as eficiências, Tabela 2. 
Figura 6 - Temperaturas para o modelo básico: (a) coletor. (b) parte quente do trocador de calor. (c) parte fria do trocador de calor.

(a)

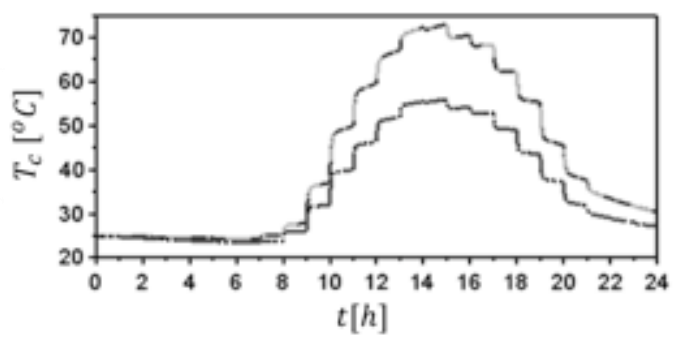

(c)
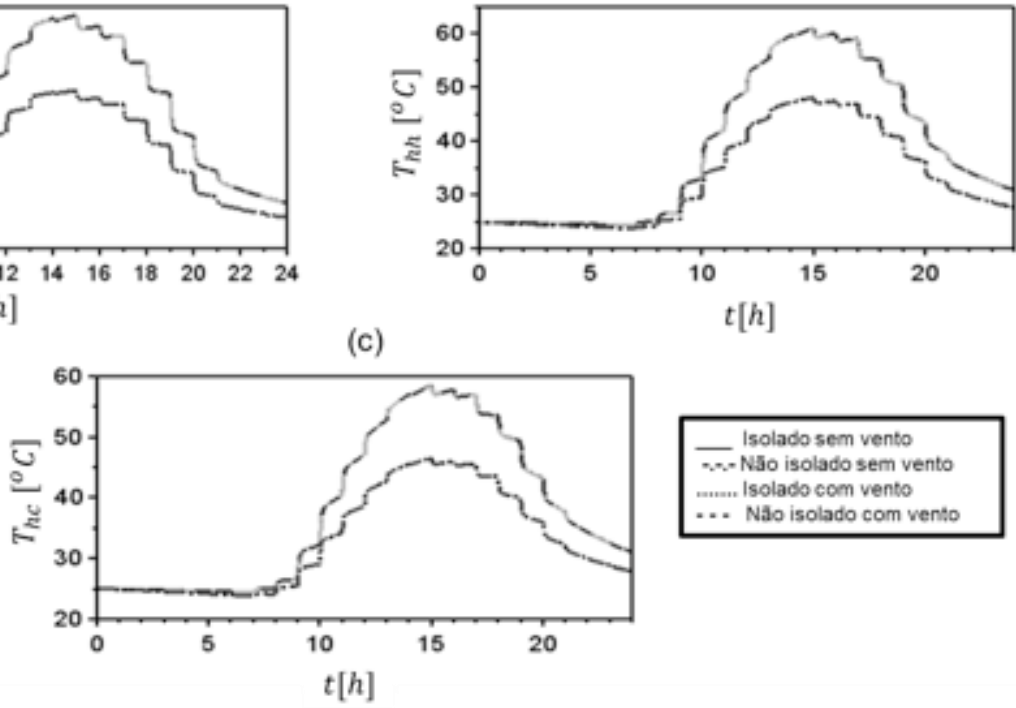

(b)

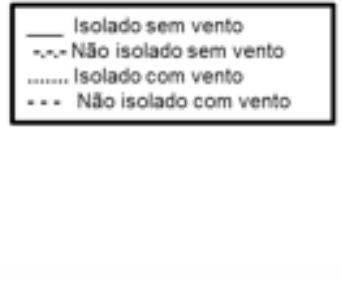

As Figuras 7 e 8 mostram os resultados das simulações para o modelo estendido com vento, sem vento, isolado e não isolado - o caso 2. Pode-se notar que o modelo isolado sem vento é o que atinge maiores temperaturas e também se mantêm quente por mais tempo.

Figura 7 - Temperaturas para o modelo estendido: (a) coletor. (b) parte fria do trocador de calor. (c) parte quente do trocador de calor.

(a)

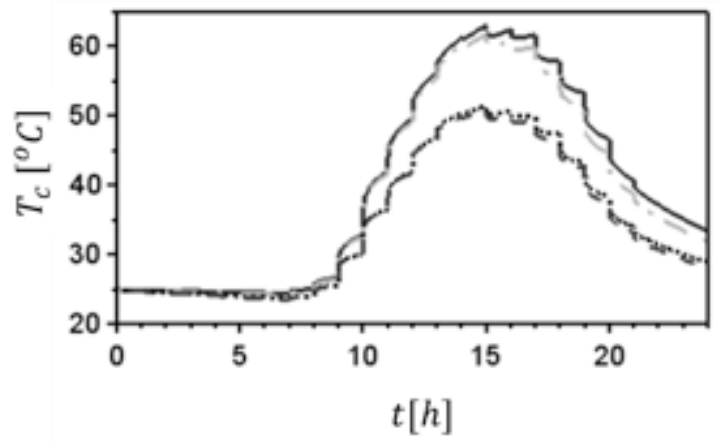

(b)

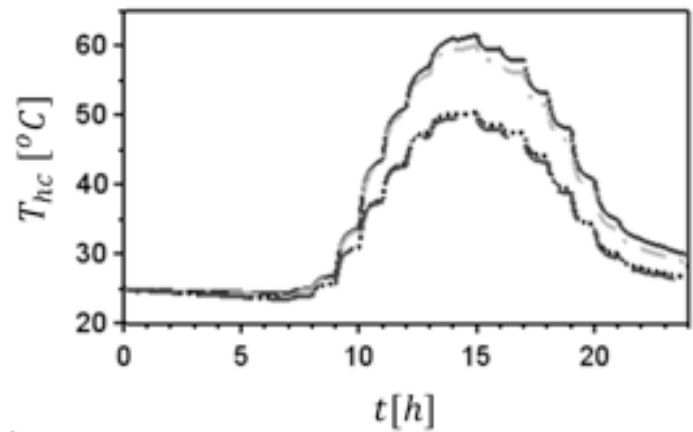

(c)

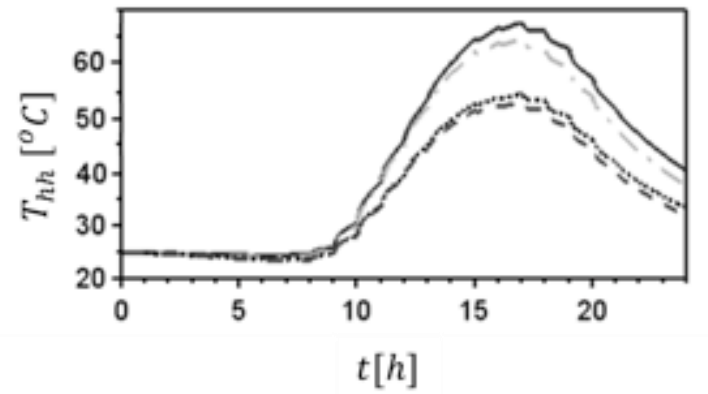

Isolado sem vento Nå isolado sem vento ..... Isolado com vento ... Não isolado com vento 
Figura 8 - Temperaturas para o modelo estendido das tubulações entre: (a) trocador de calor e coletor solar. (b) armazenador e trocador de calor.

(a)
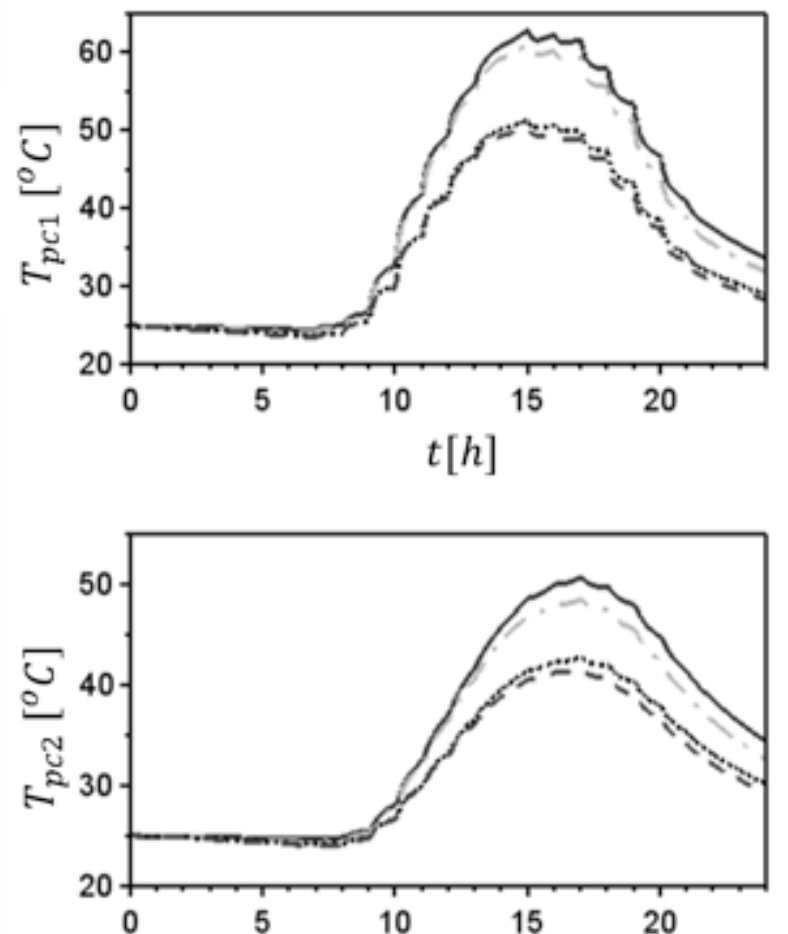

$t[h]$
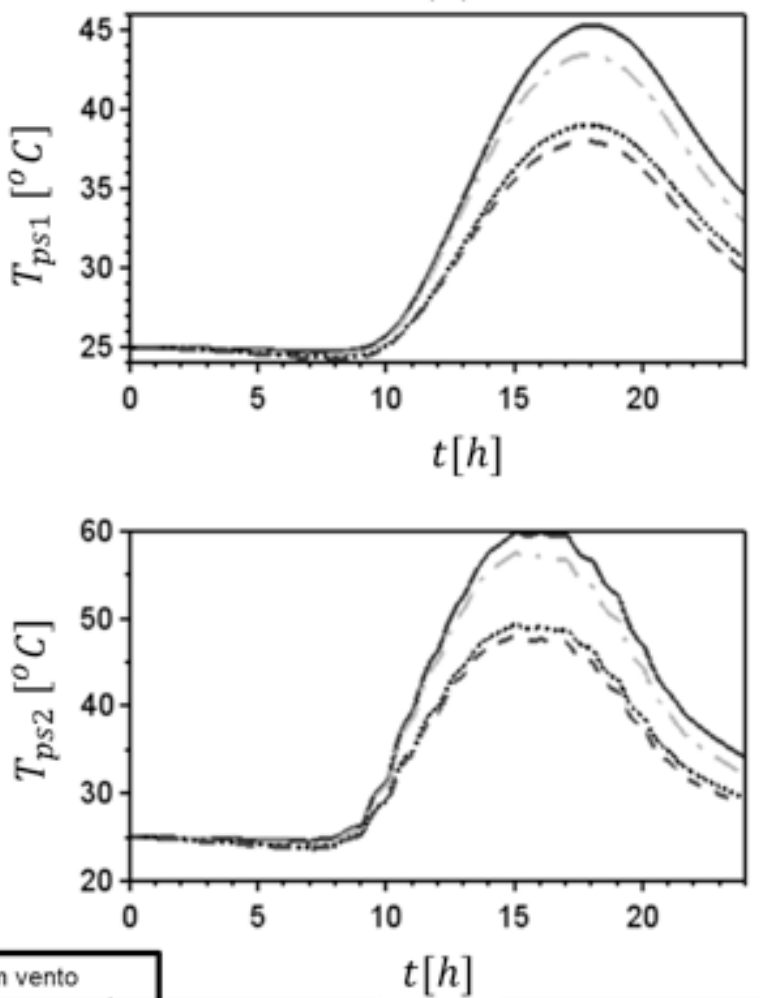

. Isolado sem vento
....- Não isolado sem vento
$\ldots . . .$. Isolado com vento
$\ldots$ Não isolado com vento

É nítida, pelas simulações do modelo estendido, a diferença quando se utiliza um isolamento para a tubulação, por mais que em termos de eficiência esta diferença não seja tão grande para o caso avaliado. Acredita-se que para sistemas reais, em que 0 campo de coletores esteja distante do local de consumo final da água aquecida esse fator será de extrema importância para se evitar perdas ao longo do processo de transporte da energia térmica absorvida.

Avaliando de forma qualitativa os resultados obtidos, quando comparado com o trabalho de Kicsiny et al. (2014), o modelo estendido foi o melhor em todos os casos estudados pelo autor, mesmo resultado encontrado neste trabalho. A temperatura máxima do coletor solar foi de aproximadamente $90^{\circ} \mathrm{C}$, em contrapartida, neste estudo, foi de aproximadamente $72^{\circ} \mathrm{C}$. Essa diferença pode ser explicada pelas condições ambientais avaliadas serem diferentes, ou seja, as condições ambientais não são passíveis de reprodução, e pelas modificações implementadas nos modelos matemáticos deste trabalho.

A avaliação do uso do isolamento e da velocidade do vento não foi implementada no trabalho de Kicsiny et al. (2014). Esse tipo de estudo foi encontrado na literatura em outros trabalhos, como o de Janjai et al. (2000), Palyvos (2008) e Spirandeli, Prado e Sousa (2017), mas para modelos matemáticos diferentes os quais levaram em consideração outros sistemas, o que dificulta a comparação. Mas vale salientar que os resultados encontrados nesses trabalhos foram semelhantes aos obtidos por este estudo, 
levando a conclusão de que o isolamento afeta a temperatura da água de forma positiva e o vento de forma negativa.

\section{CONCLUSÕES}

Os resultados obtidos pelas simulações do modelo matemático dos sistemas de aquecimento solar mostram-se eficientes para os cenários avaliados. Para ambos os casos (modelo matemático básico e estendido) a temperatura máxima atingida pela água do coletor solar foi aproximadamente $80^{\circ} \mathrm{C}$. Avaliando a temperatura do armazenador, a máxima ficou na faixa de $40^{\circ} \mathrm{C}-45^{\circ} \mathrm{C}$, temperatura suficiente para serem utilizados em sistemas para aquecimento residencial.

Através das análises da eficiência do armazenador, foi constatado que o modelo estendido foi melhor, apresentando uma eficiência de 53\%. Vale ressaltar que este representa a realidade de uma forma mais aproximada, pois leva em consideração os efeitos de transferência de calor das tubulações.

O efeito térmico da tubulação ajuda a manter a temperatura do fluido mais alta e a se manter aquecido por mais tempo, isso pode ser notado na análise das temperaturas da parte quente e parte fria do trocador de calor. $O$ fato de ser considerado isolado pode ser destacado, pois nem a tubulação do coletor nem o armazenador perdem calor para o ambiente. Assim, pode-se afirmar que a configuração do sistema afeta diretamente nos resultados simulados.

Além disso, outro fator que influencia diretamente o comportamento do sistema é o vento. Isso é observado tanto no modelo básico quanto no estendido, em que as eficiências e as temperaturas são reduzidas quando o efeito da convecção forçada é considerado.

Com a finalidade de implementar melhorias nos modelos estudados, pode-se acrescentar o efeito da influência do comprimento da tubulação na temperatura da água, para casos em que o campo de coletores está distante do ponto de consumo, como por exemplo, para piscinas aquecidas.

Ademais, outro ponto que pode ser levado em consideração no sistema é o acréscimo de outra fonte de energia, ou seja, avaliar uma configuração híbrida (energia solar + outra fonte de energia) para períodos como pouca ou nenhuma radiação solar.

\section{NOMENCLATURA}

\begin{tabular}{c|l}
\hline Parâmetro & \multicolumn{1}{c}{ Descrição } \\
\hline $\mathrm{A}\left[\mathrm{m}^{2}\right]$ & Área superficial \\
\hline $\mathrm{C}[-]$ & Constante da correlação de Nusselt \\
\hline $\mathrm{c}\left[\mathrm{Jkg} \mathrm{k}^{-1} \mathrm{~K}^{-1}\right]$ & Calor específico \\
\hline $\mathrm{E}[\mathrm{J}]$ & Energia total no volume de controle \\
\hline$\dot{\mathrm{E}}_{\mathrm{e}_{\mathrm{i}}} / \dot{\mathrm{E}}_{\mathrm{S}_{\mathrm{i}}}[\mathrm{J} / \mathrm{s}]$ & Taxa de energia que entra/sai do volume de controle \\
\hline$\dot{\mathrm{E}}_{\mathrm{g}}[\mathrm{J} / \mathrm{s}]$ & Taxa de energia gerada no volume de controle \\
\hline$\varepsilon \mathrm{k}_{\mathrm{h}}\left[W m^{-2} \mathrm{~K}^{-1}\right]$ & Coeficiente de transferência de calor dentro do trocador \\
\hline$\Phi[-]$ & Efetividade do trocador de calor. \\
\hline $\mathrm{I}_{\mathrm{c}}\left[W m^{-2}\right]$ & Irradiação solar global \\
\hline $\mathrm{k}\left[W m^{-2} \mathrm{~K}^{-1}\right]$ & Coeficiente de perda de calor \\
\hline $\mathrm{k}_{\mathrm{W}}\left[W m^{-1} K^{-1}\right]$ & Condutividade térmica do ar \\
\hline
\end{tabular}




\begin{tabular}{|c|c|}
\hline $\mathrm{L}[\mathrm{m}]$ & Comprimento \\
\hline $\mathrm{m}_{\mathrm{T}}[\mathrm{kg}]$ & Massa total no volume de controle \\
\hline$\dot{m}_{\mathrm{e}_{\mathrm{i}}} / \dot{\mathrm{m}}_{\mathrm{s}_{\mathrm{i}}}[\mathrm{kg} / \mathrm{s}]$ & Taxa mássica que entra/sai do volume de controle \\
\hline $\mathrm{m}_{\mathrm{h}}[\mathrm{kg}]$ & Massa do trocador de calor vazio \\
\hline$\eta_{0}[-]$ & Eficiência óptica do coletor \\
\hline$\rho\left[\mathrm{kgm}^{-3}\right]$ & Densidade do fluido \\
\hline $\operatorname{Pr}[-]$ & Número de Prandtl \\
\hline $\mathrm{T}[K]$ & Temperatura \\
\hline $\mathrm{U}_{\mathrm{Lb}}\left[W m^{-2} K^{-1}\right]$ & Coeficiente de perda de calor do coletor solar - modelo básico \\
\hline $\mathrm{U}_{\mathrm{Le}}\left[W m^{-2} K^{-1}\right]$ & Coeficiente de perda de calor do coletor - modelo estendido \\
\hline$v_{l}\left[m^{3} h^{-1}\right]$ & Carga de consumo do armazenador solar \\
\hline$V\left[\mathrm{~m}^{3}\right]$ & Volume do coletor solar \\
\hline$\dot{\mathrm{W}}[\mathrm{J} / \mathrm{s}]$ & Trabalho realizado/recebido pelo volume de controle \\
\hline \multicolumn{2}{|r|}{ Subscrito } \\
\hline a & Ambiente \\
\hline c & Coletor solar \\
\hline d & Água da torneira \\
\hline $\mathrm{h}$ & Trocador de calor \\
\hline $\mathrm{hc}$ & Parte fria do trocador de calor \\
\hline hh & Parte quente do trocador de calor \\
\hline $\mathrm{pc}$ & Tubulação do coletor solar \\
\hline ps & Tubulação do armazenador \\
\hline pc1 & Tubulação entre a saída do armazenador e o trocador de calor \\
\hline pc2 & Tubulação entre o trocador de calor e a entrada do armazenador \\
\hline ps1 & Tubulação entre a saída do armazenador e o trocador de calor \\
\hline ps2 & Tubulação entre o trocador de calor e a entrada do armazenador \\
\hline $\mathrm{s}$ & Armazenador \\
\hline
\end{tabular}

\section{REFERÊNCIAS}

CEMIG, Companhia Energética de Minas Gerais. Alternativas Energéticas: Uma visão CEMIG. Belo Horizonte, 2012. Disponível em: https://www.solenerg.com.br/wpcontent/uploads/2013/04/Alternativas-Energéticas-Uma-Visao-Cemig.pdf

\section{DACOL, A. P. de O. Modelagem matemática do uso da energia solar para} aquecimento de uma casa. Juína, 2012.

\section{DASOL-ABRAVA, DEPARTAMENTO NACIONAL DE AQUECIMENTO SOLAR - ASSOCIAÇÃO BRASILEIRA DE REFRIGERAÇÃO, AR CONDICIONADO, VENTILAÇÃO E AQUECIMENTO. Benefícios do Aquecimento Solar. Disponível em: <http://www.dasolabrava.org.br/informacoes/beneficios-do-aquecimento-solar/>. Acesso em: 22 mai. 2015.}

FLECK, L.; TAVARES, M. H. F.; EYNG, E. Conceitos e importância da modelagem matemática de qualidade da água para gestão dos recursos hídricos. Ambiência Revista do Setor de ciências agrárias e ambientais, v. 9, n. 3, p. 487-503, 2013. DOI: 
10.5935/ambiencia.2013.03.03 Disponível em:

https://revistas.unicentro.br/index.php/ambiencia/article/viewFile/2086/61 Acesso em: 23 abr. 2019

GOMES FILHO, C. A. G.; Análise preliminar para a estimativa da perda de calor sensível de um fluido homogêneo térmico em um reservatório de geometria cilíndrica. Trabalho de conclusão de curso (Bacharelado - Engenharia Mecânica) - Universidade Estadual Paulista, Faculdade de Engenharia de Guaratinguetá, 2013. Disponível em: https://repositorio.unesp.br/handle/11449/119280?locale-attribute=es Acesso em: 23 abr. 2019

INCROPERA, F. P.; DEWITT, D. P.; BERGMAN, T. L.; LAVINE, A. S. Fundamentos de Transferência de Calor e de Massa. 6. ed. Rio de Janeiro: LTC, 2011.

INMET, Instituto Nacional de Meteorologia. Disponível em:

<http://www.inmet.gov.br/portal/>. Acessado em: 25 mar. 2015.

JANJAI, S.; ESPER, A.; MÜHLBAUER, W. Modelling the performance of a large area plastic solar collector. Renewable Energy, Stuttgart, v. 21, p. 363-376, 2000.

KICSINY, R.; VARGA, Z. Real-time state observer design for solar termal heating systems. Applied Mathematics and Computation, v. 218, p. 11558-11569, 2012.

KICSINY. R.; NAGY. J.; SZALÓKI. C.S. Extended Ordinary Differential Equation Models for Solar Heating Systems with Pipes. Applied Energy, v. 129, p. 166-176, 2014.

PALYVOS, J. A.; A survey of wind convection coefficient correlations for building envelope energy systems' modeling. Applied Thermal Engineering, v. 28, p. 801-808, 2008.

PEREIRA, E. B.; MARTINS, F. R.; ABREU, S. L. de; RÜTHER, R. Atlas Brasileiro de Energia Solar. 1. ed. São José dos Campos, 2006.

REJOWSKI JUNIOR, R., As Novas Fronteiras e os Desafios da Simulação de Processos Químicos, Revista Brasileira de Engenharia Química, v. 34 - nํ1, p. 7-11, 2018.

RUSSI, M. Projeto e análise da eficiência de um sistema solar misto de aquecimento de água e de condicionamento térmico de edificações para Santa Maria - RS. Santa Maria, 2012. Dissertação de mestrado.

SÃO PAULO. Lei no 14.459, de 3 de Julho de 2007. Artigos $2^{\circ}$ e $3^{\circ}$. Prefeitura do Município de São Paulo, São Paulo, 3 jul. 2007. Disponível em: < http://www3.prefeitura.sp.gov.br/cadlem/secretarias/negocios_juridicos/cadlem/integra.asp ?alt=04072007L\%20144590000 >. Acesso em: 24 mai. 2015.

SECCHI, A.R., Modelagem e Simulação de Processos, Departamento de Engenharia Química, Escola de Engenharia, Universidade Federal do Rio Grande do Sul, 2002. 
SPIRANDELI, A. B. L.; PRADO, G. O.; SOUSA, N.G. Desenvolvimento de um destilador solar tipo escada e análise de desempenho em relação a um destilador solar com cobertura piramidal. XII Congresso Brasileiro de Engenharia Química em Iniciação Científica, UFSCar - São Carlos - SP, 2017.

WANG, Z.; YANG, W.; QIU, F.; ZHANG, X.; ZHAO, X. Solar water heating: From theory, application, marketing and research. Renewable and Sustainable Energy Reviews, Guangzhou, v. 41, p. 68 - 84, 2015.

Recebido em: 26/10/2018

Aprovado em: 16/04/2019 